\title{
Template-Free Synthesis of Organically Modified Silica Mesoporous Thin Films for TNT Sensing
}

\author{
Adem Yildirim, ${ }^{\dagger}$ Hulya Budunoglu, ${ }^{\dagger}$ Hakan Deniz, ${ }^{\dagger}$ Mustafa O. Guler, ${ }^{\dagger}$ and \\ Mehmet Bayindir*, $\uparrow, \neq$
}

UNAM-Institute of Materials Science and Nanotechnology and Department of Physics, Bilkent University, 06800 Ankara, Turkey

\begin{abstract}
In this paper, we present a facile, template-free sol-gel method to produce fluorescent and highly mesoporous organically modified silica (ORMOSIL) thin films for vapor phase sensing of TNT. An alkyltrifunctional, methyltrimethoxysilane MTMS precursor was used to impart hydrophobic behavior to gel network in order to form the spring back effect. In this way, porous films (up to $74 \%$ porosity) are obtained at ambient conditions. Fluorescent molecules are physically encapsulated in the ORMOSIL network during gelation. Fluorescence of the films was found to be stable even after 3 months, proving the successful fixing of the dye into the ORMOSIL network. The functional ORMOSIL thin films exhibited high fluorescence quenching upon exposition to TNT and DNT vapor. Fluorescence quenching responses of the films are thickness-dependent and higher fluorescence quenching efficiency was observed for the thinnest film (8.6\% in $10 \mathrm{~s})$. The prepared mesoporous ORMOSIL thin films have great potential in new sensor and catalysis applications.
\end{abstract}

KEYWORDS: ORMOSIL • mesoporous materials $\bullet$ fluorescence quenching $\bullet$ thin films $\bullet$ TNT detection

\section{INTRODUCTION}

M ild conditions of the sol-gel process enable mixing organic and inorganic components in the same matrix at the nanometer scale (1-3). A common example of these hybrid materials is the mesoporous silica hybrids, also known as organically modified silica (ORMOSIL), which combine high porosity with organic functionality $(4,5)$. Organic functionality is introduced into the inorganic silica matrix by one pot or grafting method; and the interaction between the components can be either with physical interactions or chemical bonds (6). It is also possible to adjust the porosity of these ORMOSILs by controlling the sol-gel process parameters, which is another related research area with potential applications in chemical sensors, catalysis, separation and dielectric materials $(7-11)$.

Producing mesoporous ORMOSIL materials as thin films enables exploiting the intrinsic properties of thin films such as better transparency, control of thickness and composition, and deposition as multilayer films (4). These mesoporous ORMOSIL thin films are generally prepared by respective hydrolysis and condensation of silicon-alkoxy precursors in the presence of surfactant molecules to form a viscous solution (sol), followed by thin film deposition, by means of standard methods such as dip-coating or spin-coating. Surfactant molecules form self-assembled templates around

\footnotetext{
* Corresponding author.Tel: +90 312 2903500. Fax: +90 3122664365 . E-mail bayindir@nano.org.tr.

Received for review June 30, 2010 and accepted August 23, 2010

† UNAM-Institute of Materials Science and Nanotechnology, Bilkent University

* Department of Physics, Bilkent University.

DOI: $10.1021 / \mathrm{am} 100568 \mathrm{c}$
}

2010 American Chemical Society which silica precursor polymerizes to form the porous network. Following complete polymerization and aging of the silica network, surfactant molecules are removed by dissolving in acidic solutions or by calcination. The end product material has well-defined pore characteristics; where pore size $(2-30 \mathrm{~nm})$ and morphology (wormlike, hexagonal, etc.) can be tuned by changing the surfactant molecule or its concentration.

Hybrid aerogel (super critically dried) and aerogel-like xerogel (ambient dried) thin films are contending alternatives to template synthesized ORMOSIL hybrids with their template-free synthesis. These thin films are intrinsically porous, with random, well-accessible, cylindrical, and branched mesopores, and their porosities are higher than the template synthesized counterparts (around 90\%) $(12,13)$. The extremely high porosity allows molecules to easily access the inner parts of the material resulting in very high diffusion rates (8). Although such high-porosity hybrid structures would be indispensable in sensing and catalysis application, there are only a few examples of such structures like transparent monoliths and thin films in these fields (14-16). Also, aerogel thin film related works have been limited to applications that use only the intrinsic properties of these materials, such as low dielectric materials and resistivity based humidity sensors, (17-22). To the best of our knowledge, this work is the first demonstration of hybrid silica aerogel thin film preparation for an advanced application like chemical sensing. Here, we report a one-pot approach for the preparation of a hybrid aerogel like xerogel thin film for TNT sensing by organic dye incorporation into ORMOSIL network. Previously, silica aerogel and aerogel like xerogel 
thin films were prepared by super critical drying but also using ambient pressure drying (APD), simplifying the overall procedure and lowering the costs. For example, Prekash et al. (17) prepared aerogel thin films by modifying the gel network with hydrophobic groups. These hydrophobic groups on the surface facilitate APD method because hydrophobic interactions prevent network collapse because of capillary tension and condensation reactions. During the gel solvent evaporation, the network first shrinks then recoils to its initial porous state, known as the "spring back" effect. In our case, we used intrinsically hydrophobic methyltrimethoxysilane (MTMS) monomer to achieve spring back effect in one pot process in which the unhydrolysable methyl groups cover the gel surface making the gel hydrophobic (23-25).

Doping silica gels with organic molecules can be achieved by physical or by covalent bonding. If the organic molecules are dissolved in the monomer solvent in order to trap in the gel network during polymerization, these molecules are washed out during the washing and supercritical drying steps (13). To prevent leakage, dopants must be fixed into the silica network by covalent bonding or electrostatic forces (14-16), which further complicates the process. Our onepot approach effectively overcomes leakage problem by altogether eliminating washing and supercritical drying steps; hence fluorescent ORMOSIL coatings were successfully prepared by physical encapsulation of the dye molecules.

\section{EXPERIMENTAL SECTION}

Materials. Methanol, ammonium hydroxide, oxalic acid, hydrochloric acid, methyltrimethoxysilane, and dinitrotoluene were purchased from Sigma-Aldrich (USA). Meso-tetrakis-pcarboxy-phenylporphyrin $\left(\mathrm{TCPPH}_{2}\right)$ was purchased from Frontier Scientific (USA). All of the chemicals were used as received.

Gel Preparation. Hydrophobic gels were prepared by modifying the method presented in ref 23. The molar ratios of MTMS, methanol, water, and TCPPH${ }_{2}$ were chosen to be 1:15:4.8:1 $\times$ $10^{-6}$. In a standard procedure; $2 \mathrm{~mL}$ of MTMS, $7.8 \mathrm{~mL}$ of methanol, and $0.6 \mathrm{~mL}$ of $0.001 \mathrm{M}$ oxalic acid in water was mixed and stirred for $30 \mathrm{~min}$ at room temperature. After $24 \mathrm{~h}$ of hydrolysis, $0.8 \mathrm{~mL}$ of ammonium hydroxide in water $(11.2$ M) was added to catalyze the condensation reaction and the solution was stirred for $15 \mathrm{~min}$ at room temperature. During stirring, $0.07 \mathrm{mg}$ of $\mathrm{TCPPH}_{2}$ was dissolved in $0.5 \mathrm{~mL}$ of methanol and added to the reaction solution. The solution was then incubated at ambient conditions for 2 days for gelation and aging.

Film Preparation. A spin-coating method was used for aerogel thin film production. Before spin-coating, the aged gel was broken into small pieces and $15 \mathrm{~mL}$ of methanol was added. After that, it was sonicated with an ultrasonic homogenizer to obtain a suitable sol for spin coating. The optimum time and power for homogenization were found to be $45 \mathrm{~s}$ and $20 \mathrm{~W}$, respectively. The sol was then spin-coated on $1.5 \mathrm{~cm} \times$ $3 \mathrm{~cm}$ glass substrates with a speed of $2000 \mathrm{rpm}$. The glass substrates were sonicated in ethanol before coating. Most of the solvent evaporates during the spin-coating process. The films were dried at room temperature overnight for further solvent evaporation.

Nonporous Film Preparation. For a control experiment, a nonporous film was also prepared. In a standard procedure, 2 $\mathrm{mL}$ of MTMS, $2 \mathrm{~mL}$ of ethanol, $0.2 \mathrm{~mL}$ of water, and $5 \mu \mathrm{L}$ of $37 \%$ hydrochloric acid were mixed and stirred at $60{ }^{\circ} \mathrm{C}$ for 90 $\mathrm{min}$, and then $0.4 \mathrm{~mL} \mathrm{HCl}(0.1 \mathrm{M})$ and $0.35 \mathrm{~mL}$ water were added and the solution was further stirred for $15 \mathrm{~min}$ at room temperature. Finally, the sol was aged at $50{ }^{\circ} \mathrm{C}$ for $20 \mathrm{~min}$ and coated on glass substrates with spin-coating at $2000 \mathrm{rpm}$.

Fluorescence Quenching Experiments. The fluorescence quenching experiments were performed similar to the previous reports (26-30). Approximately $0.5 \mathrm{~g}$ of TNT or DNT were placed into a $15 \mathrm{~mL}$ vial and covered with cotton to prevent direct contact of the analyte molecules with the films and to maintain constant analyte pressure. The ORMOSIL films were exposed to TNT or DNT vapor for a specified period of time and then fluorescence measurements were carried out immediately. Measurements were performed at room temperature and it is assumed that the vial atmosphere was saturated with analyte vapor (9)

Characterization. TEM bright-field images were taken using a FEI Tecnai G2 F30 microscope operated at $200 \mathrm{kV}$. TEM samples were prepared by scratching off aerogel thin films from the surface and dispersed in ethanol. A drop of solution was placed on a holey carbon-coated copper grid. SEM images were taken with FEI Quanta 200F ESEM microscope at low vacuum conditions. UV-vis absorption and tranmission spectra were recorded by Varian Carry 100 spectrophotometer. Fluorescence spectra were recorded by Varian Eclipse spectrophotometer. Thickness and refractive index measurements were performed by using J. A. Woollam V-Vase Ellipsometer. A contact angle meter (OCA 30, Dataphysics) was used to measure the static water contact angles on the aerogel thin films. Water droplets of $0.4 \mu \mathrm{L}$ volume were used and Laplace-Young fitting was applied on contact angle measurements.

\section{RESULTS AND DISCUSSION}

Hybrid organically modified silica gels were prepared by a two-step sol-gel method. First step is hydrolysis of MTMS in the presence of methanol, water, and acid and the second step is the condensation with base. A porphyrin derivative $\left(\mathrm{TCPPH}_{2}\right)$ was directly added into the solution during condensation. $\mathrm{TCPPH}_{2}$ molecules enable TNT and DNT detection by fluorescence quenching with excited state electron transfer mechanism $(9,26)$. High porosity and high surface area of the ORMOSIL network provides fast interaction of analyte with dye molecules (Scheme 1).

In this study, three types of aerogel thin films (AF-1, AF2 , and AF-3) with different thicknesses were produced by diluting the homogenized gel with methanol (Table 1). Also a nonporous xerogel film (NF) was prepared for control experiments. Average thicknesses and refractive indices of the films were determined with an ellipsometer using spectroscopic ellipsometry (reflection mode) measurements and found to be $120 \mathrm{~nm}$ for $\mathrm{AF}-3$ and $1.1 \mu \mathrm{m}$ for AF-1. As expected, average thickness of the films decreases with increasing dilution. The relationship between dilution and average film thicknesses were fairly linear. The film thickness is not only affected by the silica concentration in the sol but also affected by the viscosity of the sol. The homogenized solution was quite viscous before dilution. Diluting the sol with methanol significantly lowered the viscosity of the sol, therefore decreasing the film thicknesses more than expected (3 times diluting results 4.6 fold decrease in thickness).

Aging the gels is found to be critical for strengthening gel network and preventing pore collapse during spin coating and drying. Gels were aged for 2 days at room temperature to obtain the highly porous films. Scanning electron micro- 
Scheme 1. (a) Preparation of Fluorescent Mesoporous Thin Films; (b) Schematic Illustration of Porous Network upon TNT Exposure; (c) Schematic Illustration of TNT Sensing with $\mathrm{TCPPH}_{2}$ Dye

(a)

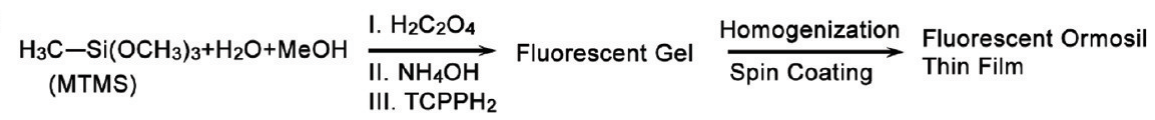

(b)

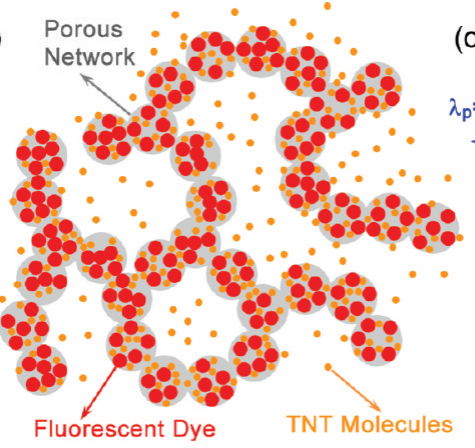

(c)

Table 1. Physical Properties of ORMOSIL Films

\begin{tabular}{lcrccc}
\hline film & $\begin{array}{c}\text { dilution } \\
(\mathrm{v} / \mathrm{v})^{a}\end{array}$ & $\begin{array}{c}\text { average } \\
\text { thickness } \\
(\mathrm{nm})^{b}\end{array}$ & $\begin{array}{c}\text { refractive } \\
\text { index }\left(n_{\mathrm{D}}\right) \\
\text { at } 650 \mathrm{~nm}\end{array}$ & $\begin{array}{c}\text { porosity } \\
(\%)\end{array}$ & $\begin{array}{c}\text { water } \\
\text { contact } \\
\text { angle }(\mathrm{deg})\end{array}$ \\
\hline $\mathrm{AF}-1$ & & $1133 \pm 1.2$ & 1.182 & 54.4 & 147.1 \\
$\mathrm{AF}-2$ & $1-3$ & $246 \pm 5.6$ & 1.139 & 65.2 & 138.3 \\
AF-3 & $1-5$ & $120 \pm 0.6$ & 1.105 & 73.7 & 120.3 \\
NF & & $292 \pm 0.8$ & 1.399 & &
\end{tabular}

${ }^{a}$ Sonicated sol is further diluted with methanol. ${ }^{b}$ Measured by spectroscopic ellipsometer.

scope (SEM) images of AF-1 reveal the aerogel thin film formation with macro and mesoporous structures (Figure 1). Domain sizes of the prepared aerogel thin films were further characterized by transmission electron microscope (TEM) and meso and macro sized pore formation were observed (Figure 2 ). Total porosity $(\pi)$ of the films were calculated by porosity refractive index equation (16)

$$
\pi=\left[\frac{1.399-n_{\mathrm{D}}}{1.399-n_{\mathrm{a}}}\right]
$$

Where 1.399 is the measured refractive index of nonporous film (NF), and $n_{\mathrm{D}}$ and $n_{\mathrm{a}}$ are the refractive indices of the porous film and air, respectively (Table 1 and Figure $3 \mathrm{~b}$ inset). At higher dilution, the porosity of the films increases because of increasing macroporosity, which is also confirmed by SEM images.

The mesoporous ORMOSIL films are intrinsically hydrophobic because of the MTMS monomer which contains a nonhydrolyzable methyl group (31). During polymerization, the methyl groups cover the surface and provide hydrophobic characteristic to the aerogel. To quantify surface energy of the aerogels, we measured static contact angles (Table 1). High contact angle $\left(147.1^{\circ}\right)$ for AF-1 film indicates that the surface is very hydrophobic. Besides the methyl groups, surface roughness also contributed to the high hydrophobicity of the films. The hydrophobicity of the surface provides extra stability against moisture in atmospheric conditions and so desirable in many thin film applications. Relatively lower contact angles observed for AF-2 (138.3 $)$ and AF-3 $\left(120.3^{\circ}\right)$ are due to the nonhomogeneous film formation. In these films, ORMOSIL particles do not cover the glass substrate completely (see the Supporting Information, Figures S1 and S2) and some parts of the glass substrate remain uncoated. These hydrophilic uncoated domains decrease the water contact angle of the film.

The bulk gels prepared by the above-described method are opaque. However, their thin-film counterparts were found to be highly transparent in the visible region, making them suitable for optical applications. Figure 3 a shows the transparency of AF-3 on glass substrate. Film transparency increases with decreasing film thickness; AF- 1 was $82 \%$ and AF-3 was $89 \%$ transparent at $650 \mathrm{~nm}$ (Figure 3b). The absorption and emission spectra of AF-1 film are shown in Figure 4. The emission spectra of $\mathrm{TCPPH}_{2}$ in ethanol and in AF-1 are identical confirming the incorporation of the dye molecules in the ORMOSIL network. Although the TCPPH molecules were physically encapsulated through the silica network, there is no loss in the fluorescence of the films even after 3 months. The optical measurements of the films prove that highly transparent ORMOSIL aerogel thin films with stable fluorescence were successfully prepared.

Fluorescence quenching was observed for all films upon exposure to TNT molecules. Figure 5 shows the quenching efficiencies of the films with respect to time. AF-3 was found to have quenching efficiencies of $8.6 \%$ in $10 \mathrm{~s}$ and $28.2 \%$ in $60 \mathrm{~s}$. This high quenching efficiency is due to high surface area and high porosity of the films. Meso- and macropores of the films enable faster diffusion of the analyte molecules, and high surface area enhances the analyte dye interaction. AF-1 demonstrated quenching efficiencies of $2.1 \%$ in $10 \mathrm{~s}$ and $\mathrm{AF}-25 \%$ in $10 \mathrm{~s}$. As expected, the nonporous film exhibited very low quenching efficiency $(<1 \%)$ for the first $10 \mathrm{~s}$. Quenching response increases with decreasing film thickness (Figure 5b). This can be attributed to the higher macroporosity in thinner films increasing surface area and thereby analyte dye interaction.

Fluorescence quenching performance of the AF-3 was inspected also with DNT and the quenching efficiency was 

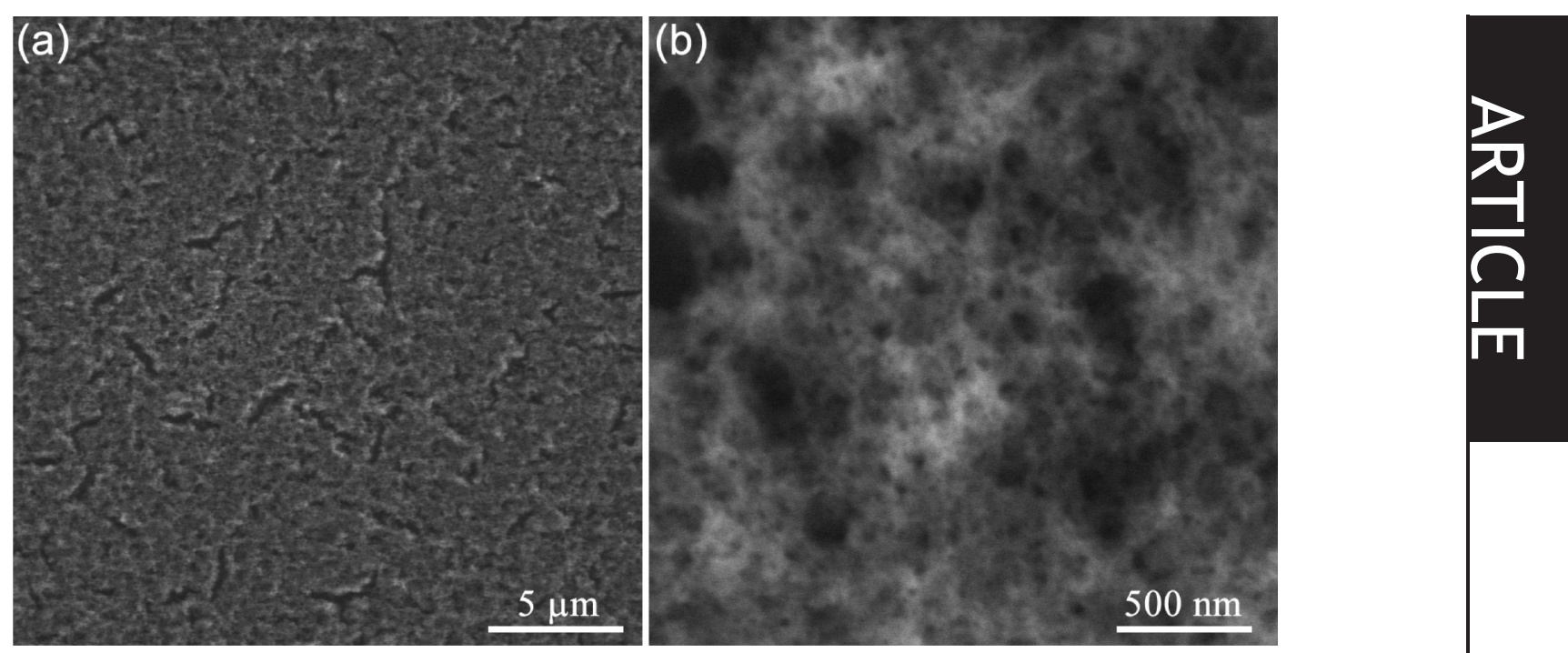

FIGURE 1. SEM micrograph of mesoporous ORMOSIL thin film (AF-1) shows (a) large area and (b) close-up region.

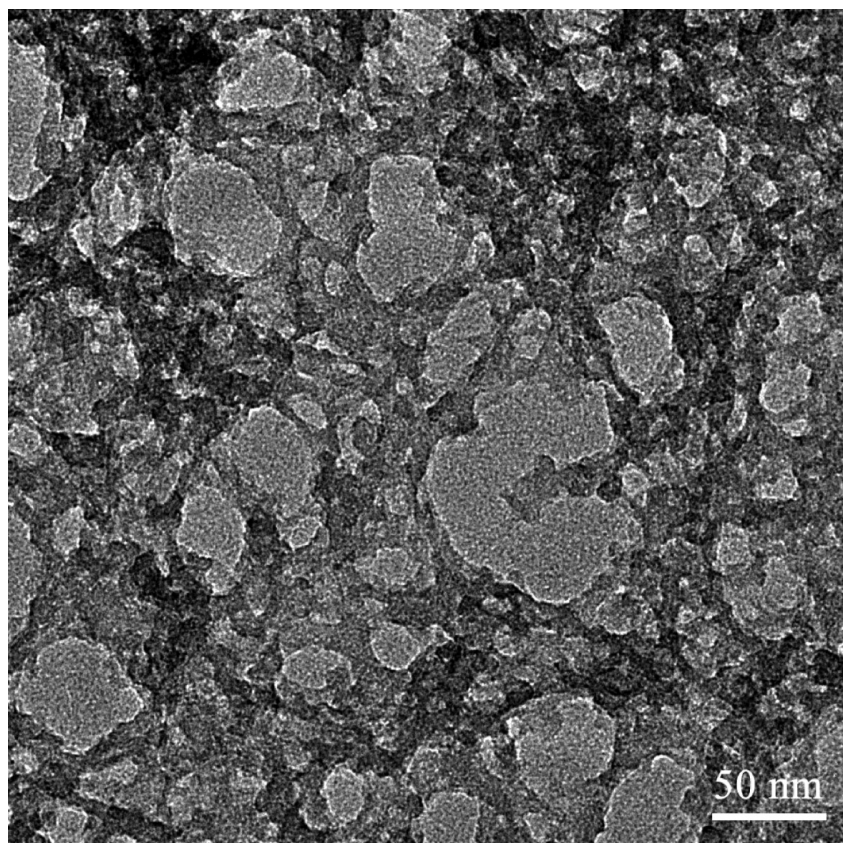

FIGURE 2. TEM micrograph of mesoporous ORMOSIL network.

found to be $10.3 \%$ in $10 \mathrm{~s}$ and $20.6 \%$ in $60 \mathrm{~s}$ (Figure 6a). DNT quenching efficiency was approximately equal to the quenching efficiency of TNT in the first minute. However, after the first minute, TNT quenching efficiency increases remarkably compared to DNT (Figure 6b) although DNT has higher vapor pressure (180 ppb) than TNT (10 ppb) at RT. It is known that the fluorescence quenching efficiency of an analyte is not only affected by its vapor pressure, but also by the exergonicity $\left(-c \mathrm{G}^{\circ}\right)$ of electron transfer between dye and analyte and the binding strength of the analyte. The binding constant of TNT to electron-rich porphyrin ring is higher than DNT because of its extra electron-withdrawing nitro group (27), hence similar fluorescence quenching responses are observed for DNT and TNT. Also, it must be noted that because of the high binding strength of TNT to porphyrin dyes, the fluorescence recovery is very slow. We
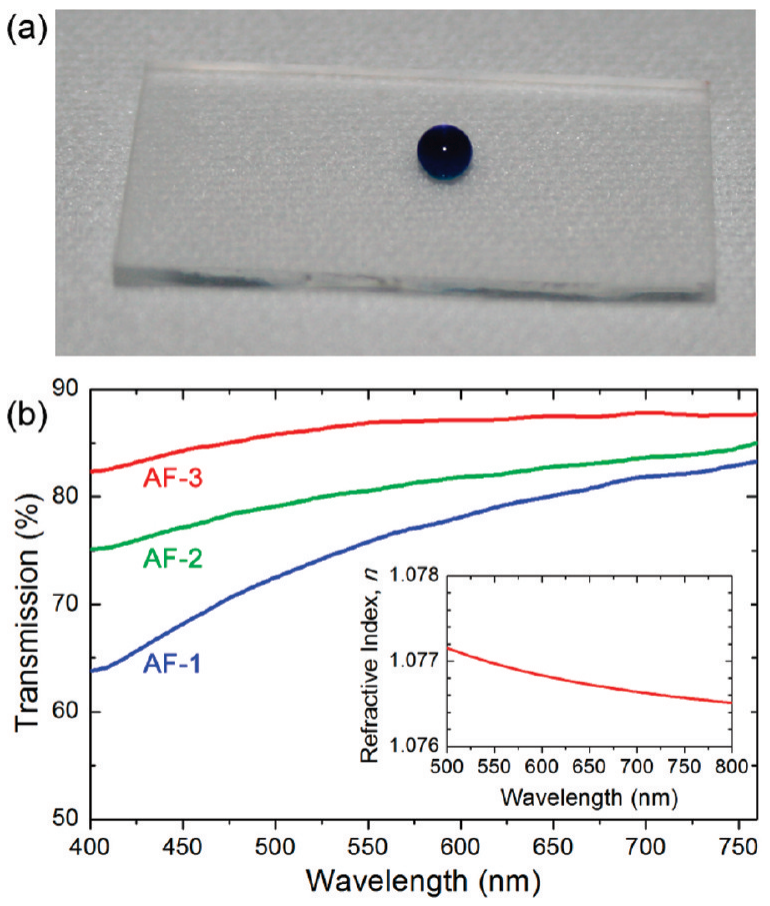

FIGURE 3. (a) Photograph of the water droplet on highly transparent AF-1. (b) Transmission spectra of films in visible range. The inset shows measured refractive index of AF-3 as a function of wavelength by spectrometric ellipsometer.

observed that even after 1 day, there was no fluorescence recovery in the films.

Conjugated polymers (26-30) and porphyrin-doped mesoporous silicas $(9,26)$ are the two of the most commonly used materials in fluorescence quenching-based explosive detection. Conjugated polymer based explosive sensors are generally used as very thin layers which reduces the fluorescence intensity. In this paper, we prepared a sufficiently thick film (AF-3, $\sim 120 \mathrm{~nm}$ ) to produce measurable and reproducible fluorescence signals. The conjugated polymer sensors also suffer from photobleaching whereas the inert silica matrix in fluorescent ORMOSIL aerogel films provides photochemical stability to the $\mathrm{TCPPH}_{2}$ dye at atmospheric conditions. Dye-doped mesoporous silica films are also 


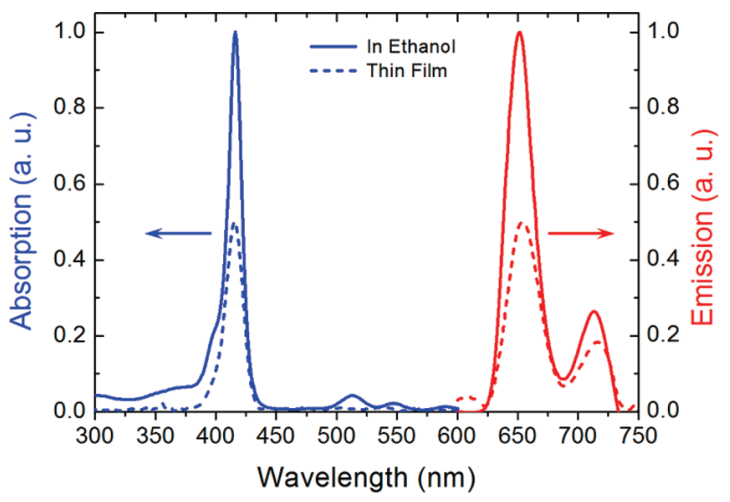

FIGURE 4. Absorption (blue) and emission (red) spectra of $\mathrm{TCPPH}_{2}$ in ethanol and in ORMOSIL thin film.

(a)

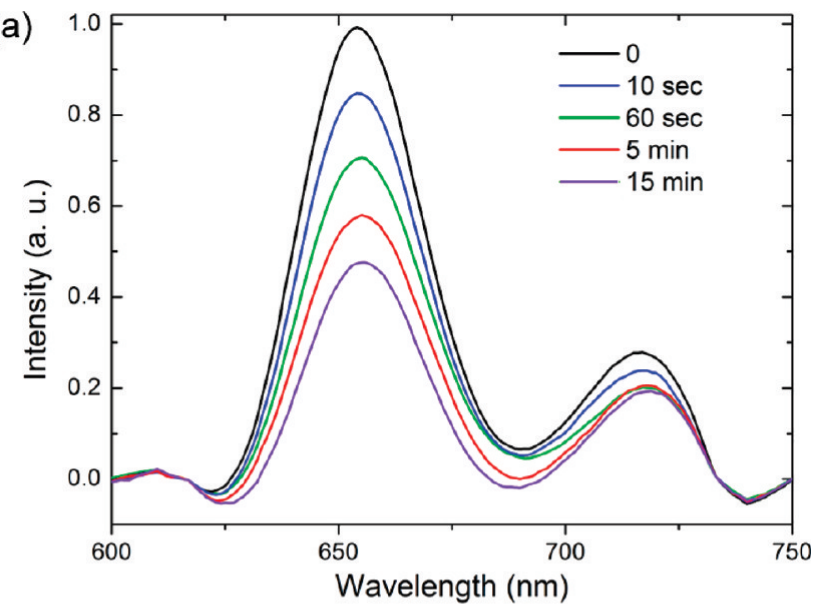

(b)

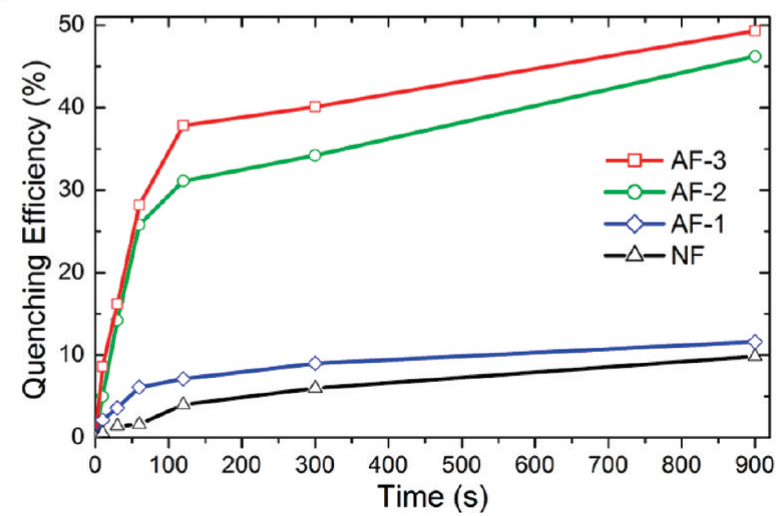

FIGURE 5. (a) Fluorescence quenching of AF-3 with TNT. (b) Quenching efficiencies of films with respect to time.

photochemically stable (9). However, the surfactant removal step can often collapse the mesopores decreasing the reproducibility of the films. Another concern about mesoporous silica films is that they are not stable under atmospheric conditions because of their hydrophilicity, which results in pore collapse due to capillary stress exerted by absorbed water. Quenching efficiencies of the ORMOSIL aerogel thin films (around \% 10) are lower than the both conjugated polymer and mesoporous silica films (for both around $30 \%$ ), but it is still enough to produce measurable fluorescence quenching response in $10 \mathrm{~s}$. In this work, hydrophobic ORMOSIL mesoporous thin films were produced using a (a)

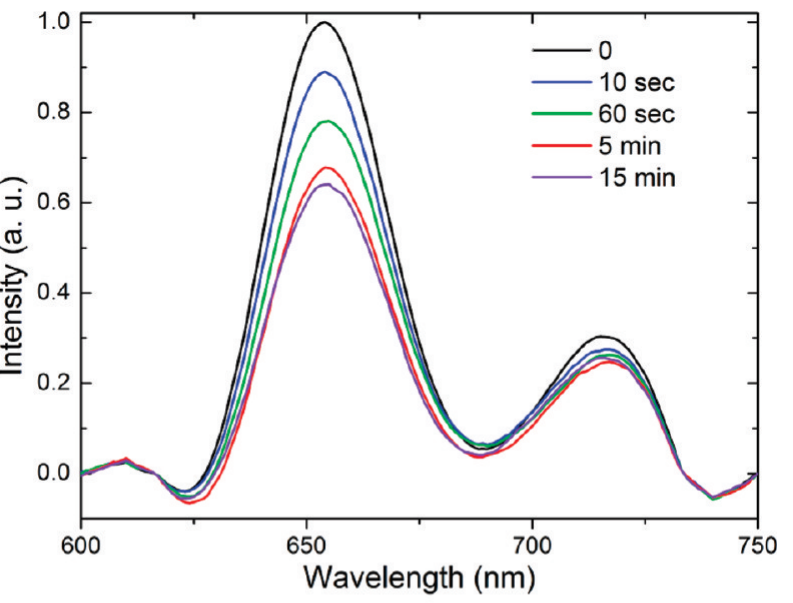

(b)

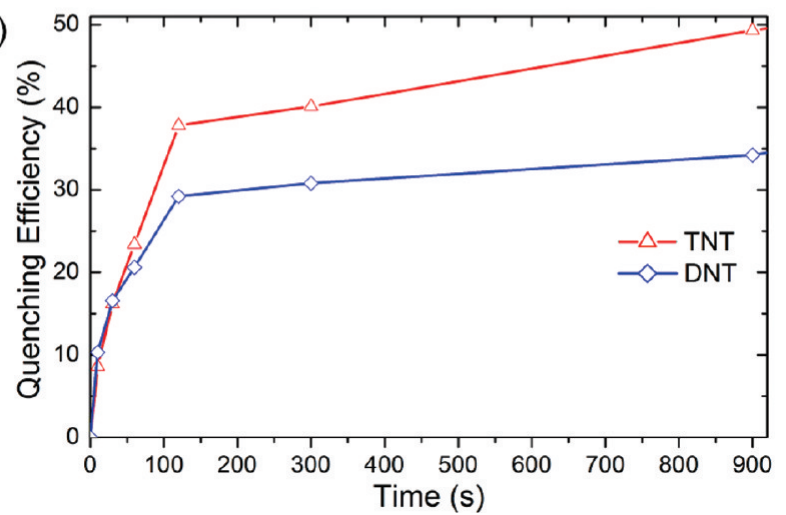

FIGURE 6. (a) Fluorescence quenching of AF-3 with DNT. (b) Quenching efficiencies of films with respect to time.

template-free method with a fluorescence quenching efficiency comparable with the contending alternatives.

\section{CONCLUSIONS}

In summary, highly porous and transparent ORMOSIL aerogel films with stable fluorescence properties were prepared for gaseous phase TNT and DNT detection. Maintaining a porous gel network during solvent evaporation at ambient conditions was simply achieved by using methyltrimethoxysilane (MTMS) monomer. MTMS provides hydrophobic behavior to the gel network that forms spring back effect and prevents pore collapse. A porphyrin derivative $\mathrm{TCPPH}_{2}$ was used as a fluorescent dopant without any chemical modification for covalent linking. Fluorescence of the films was found to be stable even after several months, proving the successful fixing of the dye into the silica network. The fluorescence quenching efficiencies against nitro-explosives were tested with TNT and DNT. All of the films exhibited fluorescence quenching with TNT and DNT. The quenching efficiency strongly depends on the film thickness. Highest quenching efficiency against TNT was observed for the thinnest film, (AF-3, average thickness is $120 \mathrm{~nm}) 8.6 \%$ in $10 \mathrm{~s}$ and $28.2 \%$ in $60 \mathrm{~s}$. Produced fluorescent ORMOSIL aerogel films are expected to have potential use in sensor applications, catalysis, and LEDs because of their easy and fast production and physicochemical stability. 
Acknowledgment. We thank Mecit Yaman for fruitful discussions. This work is supported by TÜBITAK under Project 106G090. M.B. acknowledges support from the Turkish Academy of Sciences Distinguished Young Scientist Award (TUBA GEBIP). This work was performed at UNAMInstitute of Materials Science and Nanotechnology supported by the State Planning Organization of Turkey through the National Nanotechnology Research Center Project. We also thank Turkish National Police Intelligence Department for their support.

Supporting Information Available: Chart summarizing the experimental procedures, scheme explaining the film formation and SEM images (PDF). This material is available free of charge via the Internet at http://pubs.acs.org.

\section{REFERENCES AND NOTES}

(1) Sanchez, C.; Lebeau, B.; Chaput, F.; Boilot, J. P. Adv. Mater. 2003, 15,1969 .

(2) Soler-Illia, G. J. A. A.; Innocenzi, P. Chem.-Eur.J. 2006, 12, 4478.

(3) Schottner, G. Chem. Mater. 2001, 13, 3422.

(4) Nicole, L.; Boissiere, C.; Grosso, D.; Quach, A.; Sanchez, C. J. Mater. Chem. 2005, 15, 3598.

(5) Hoffmann, F.; Cornelius, M.; Morell, J.; Fröba, M. Angew. Chem., Int. Ed. 2006, 45, 3216.

(6) Soler-Illia, G. J. A. A.; Sanchez, C.; Lebeau, B.; Patarin, J. Chem. Rev. 2002, 102, 4093.

(7) Asefa, T.; MacLachlan, M. J.; Coombs, N.; Ozin, G. A. Nature 1999, 402, 867

(8) Bonnet, M.; Schmid, L.; Baiker, A.; Diederich, F. Adv. Funct. Mater. 2002, 12, 39

(9) Tao, S.; Li, G.; Zhu, H. J.Mater. Chem. 2006, 16, 4521.

(10) Inagaki, S.; Guan, S.; Ohsuna, T.; Terasaki, O. Nature 2002, 416, 304.
(11) Mal, N. K.; Fujiwara, M.; Tanaka, Y. Nature 2003, 421, 350.

(12) Hüsing, N.; Schubert, U. Angew. Chem., Int. Ed. 1998, 37, 23.

(13) Pierre, A. C.; Pajonk, G. M. Chem. Rev. 2002, 102, 4243.

(14) Leventis, N.; Elder, I. A. Chem. Mater. 1999, 11, 2837.

(15) Rodenbusch, F. S.; Campo, L. F.; Rigacci, S.; Stefani, V. Macromol. Symp. 2005, 229, 188.

(16) Leventis, N.; Rawashdeh, A. M.; Elder, I. A.; Yang, J.; Dass, A.; Leventis, C. Chem. Mater. 2004, 16, 1493.

(17) Prakash, S. S.; Brinker, C. J.; Hurd, A. J.; Rao, S. M. Nature 1995, $374,439$.

(18) Yang, H. S.; Choi, S. Y.; Hyun, S. H.; Park, H. H.; Hong, J. K. J.NonCryst Solid. 1997, 221, 151.

(19) Kim, G. S.; Hyun, S. H. Thin Solid Films 2004, 460, 190.

(20) Girona, M. M.; Roig, A.; Benito, M.; Molins, E. J.Mater. Chem. 2003, 13, 2066

(21) Wang, C. T.; Wu, C. L.; Chen, I. C.; Huang, Y. H. Sens. Actuators B 2005, 107, 402 .

(22) Barkhudarov, P. M.; Shah, P. B.; Watkins, E. B.; Doshi, D. A.; Brinker, C. J.; Majewski, J. Corros. Sci. 2008, 50, 897.

(23) Bhagat, S. D.; Oh, C. S.; Kim, Y. H.; Ahn, Y. S.; Yeo, J. G. Microporous Mesoporous Mater. 2007, 100, 350.

(24) Rao, A. V.; Kulkarni, M. M.; Amalnerkar, D. P.; Seth, T. J.NonCryst Solid. 2003, 330, 187

(25) Budunoglu, H.; Yildirim, A.; Guler, M. O.; Bayindir, M. Manuscript in preparation.

(26) Tao, S.; Shi, Z.; Li, G.; Li, P. Chem. Phys. Chem. 2006, 7, 1902.

(27) Yang, J. S.; Swager, T. M. J. Am. Chem. Soc. 1998, 120, 11864.

(28) McQuade, D. T.; Pullen, A. E.; Swager, T. M. Chem. Rev. 2000, $100,2537$.

(29) Rose, A.; Zhu, Z.; Madigan, C. F.; Swager, T. M.; Bulovic, V. Nature 2005, 434, 876 .

(30) Toal, S. J.; Trogler, W. C. J. Mater. Chem. 2006, 16, 2871.

(31) Martin, L.; Osso, J. O.; Ricart, S.; Roig, A.; Garcia, O.; Sastre, R. J. Mater. Chem. 2008, 18, 207.

AM1 00568C 